\title{
Standards in Dermatologic Imaging
}

Ashfaq A. Marghoob, MD; for the International Skin Imaging Collaboration Melanoma Project Working Groups

The current era of ubiquitous digital cameras, digital cameras integrated into smartphones, and virtually limitless data storage affords exciting new opportunities for medicine in general and specifically dermatol$\leftarrow$ ogy. Digital photography has the potential to dramatically enable and facilitate improvements in dermatology teaching, clinical documentation, and diagnosis. One of the barriers to the diffusion of digital imaging into dermatology practice is the lack of standards for digital photography. As noted in the article by Quigley et al, ${ }^{1}$ there are currently no standards for dermatologic photography designated by Digital Imaging and Communications in Medicine. While some organizations, such as the American Teledermatology Association, ${ }^{2}$ have offered general guidelines, to our knowledge, no consistent actionable standards exist in medical publications. The absence of standards severely impedes the integration of dermatologic images across systems that support documentation, diagnosis, and clinical practice.

In a white paper for the Digital Imaging and Communications in Medicine working group, Madden ${ }^{3}$ enumerates the many challenges to the development and implementation of dermatologic imaging standards. Foremost among these is the typical reliance on consumer off-the-shelf cameras and monitors for dermatologic imaging. Off-the-shelf technologies are designed for consumer satisfaction rather than optimization of clinically relevant imaging. The image processing algorithms, white balance, and color calibration used by these cameras and monitors are inherently proprietary and continuously changing. In addition to introducing technical limitations and variability, reliance on off-the-shelf technology has impeded the establishment of a significant capital equipment market in dermatologic imaging. As such, there are no vendors who are motivated to invest heavily in the development and validation of dermatology-relevant standards across the entire spectrum of image acquisition, storage, and display.

Today, patients routinely use "selfies" to communicate with their physicians about lesions they are concerned about, and they are also beginning to use selfies as a means for monitoring nevi, a variety of skin eruptions, and ulcers. In addition, the widespread use of smartphones has spawned a plethora of dermatology applications (“apps”), with new releases being added to the ever-expanding apps list on a regular basis. Unfortunately, while the public has been quick to adopt these trends, many dermatologists are still documenting their findings via text messaging. However, basic standards could go a long way toward improving the utility of dermatologic photography. While adequate image quality may be subjective and differ by the application (eg, diagnosis vs docu- mentation), a standard approach to image-associated metadata is needed to have interchangeable systems and methods for filtering images for quality assurance and control. A prerequisite for consistent metadata is consistent terminology for technical (eg, image type) and clinical (eg, anatomic site) data. The common terminology should be aligned as closely as possible with existing standards, such as Systematized Nomenclature of Medicine Clinical Terms ${ }^{4}$ and Institute of Electrical and Electronics Engineers standards. ${ }^{5}$

In the absence of external regulatory and industry drivers of dermatology imaging standards, it behooves the dermatology community to work toward the establishment and diffusion of some basic standards. Failing to do so creates the risk of missing out on the full benefits of imaging for our patients and reflects adversely on our profession. Despite much resistance from physicians, it is clear that the electronic medical record is permeating medical practice owing to requirements set forth by federal and private health care payers. In the process of incorporating a text-based and metadata-based documentation system, considering the need to properly reflect the elements of an encounter to support the coding for a given visit, dermatologists and other medical professionals spend a significant portion of their daily clinical effort in meeting these expectations. Unfortunately, standards to incorporate the valuable presence of digital images into the electronic medical record system do not have the same level of interest and adoption. The accuracy of dermatological findings documented through digital images far exceeds what even the most detailed text can reflect. Establishing a consistent approach to image acquisition, storage, and viewing has the potential to preserve, in the long term, the most valuable aspect of a medical specialty that is based on visual assessment of the human skin. If the dermatology community fails to take a leadership role in defining and setting imaging standards, there is a risk that impractical or specialty-inappropriate standards will be imposed by external organizations.

An obvious approach for dermatology as a specialty is to leverage the effort already set forth by established medical communities. A series of radiologic standards on medical imaging as it relates to storage, security, and viewing has already been established and could serve as a platform for skin-related imaging standardization. Within dermatology, established communities with shared clinical or research goals are best suited to contribute to the evolution of standards. One such community is the melanoma-dermoscopy community, a collegial group with an impressive record of broad-based collaborative research. ${ }^{6}$ In addition, an associated cottage industry of vendors of imaging technology has arisen around this community. This community has established the International Skin Imaging Collaboration (ISIC) under the auspices of the International So- 
ciety for Digital Imaging of the Skin to address the unmet need for digital-imaging standards. ${ }^{7}$ The initial endeavor of ISIC is the Melanoma Project, a collaborative effort between skin cancer specialists (International Dermoscopy Society), informatics experts (IBM), and imaging technology developers (manufactures of dermoscopes, cameras, lenses, and skin imaging systems). The overarching goal of the Melanoma Project is to support efforts to reduce melanoma-related deaths and unnecessary biopsies by improving the accuracy and efficiency of early detection of melanoma. ${ }^{7}$ This project consists of the development of skin imaging standards and the creation of a public archive of annotated and validated skin lesion images.

The optimal use of images for education, self-diagnosis, telediagnosis, computer-assisted diagnosis, patient triage, mole monitoring, documentation, and coordination of care will benefit from the development of technology, techniques, and terminology standards. With this in mind, ISIC has created 3 working groups charged with defining and creating a set of standards. The Technology Working Group's responsibility is to develop standards for minimum functional capabilities for the equipment and settings used in the acquisition, processing, compression, display, printing, archiving, retrieval, and storage systems and to secure the transmission of clinical and dermoscopic images. The Technique Working Group's responsibility is to develop standards specifying minimum requirements for physical conditions that may significantly affect the quality and consistency of clinical images (lighting, background color, camera position, equipment calibration and maintenance, image metadata, standard patient poses, and lesion magnification). Standards for obtaining patient consent, protecting patient privacy with respect to image use, and preventing unauthorized access to patient images also fall within the scope of standards assigned to this working group. Last, the Terminology Working Group's responsibility is to develop a lexicon of standardized terms to describe anatomical sites, clinical features, dermoscopic characteristics, and diagnoses of skin lesions.

Currently, efforts in education and diagnosis regarding melanoma typically rely on convenience sampling of a limited number of images that vary in annotation, quality, and technique. A large public repository of skin images that meet the standards developed by the ISIC Melanoma Project can serve multiple communities. Physicians and educators can use the archive to improve diagnostic skills, conduct research, and provide clinical support in the identification of skin lesions that display unusual morphological characteristics. The general public may use the archive for self-education about melanoma and the benefits of early detection. Finally, developers of diagnostic equipment for skin lesions may test new devices using the standard reference images in the archive. Based on the aforementioned issues, the second part of the Melanoma Project is designed to establish a public open-access image archive. Skin lesion images that are to be used only as a reference and that are suitable for inclusion in this "Public Skin Lesion Image Archive" will be annotated using standards defined by the ISIC working groups. In addition, ISIC has successfully collaborated with numerous computer programmers, including programmers at IBM, on the development of a set of image-analysis tools (eg, lesion segmentation) and semiautomated annotation and mark-up tools. These tools will not only be useful in the acquisition and annotation of images for the public image archive but will serve as benchmarks for others who are interested in developing image-analysis programs for skin lesions.

As is evident in the review by Quigley et $\mathrm{al}^{1}$ in this issue, there is a glaring lack of standards regarding skin-lesion imaging. Before outside agencies impose standards on the dermatology community, it is imperative that we act quickly and effectively by creating and defining the imaging standards appropriate for our specialty.

\section{ARTICLE INFORMATION}

Author Affiliation: Dermatology Service, Memorial Sloan Kettering Cancer Center, Hauppauge, New York.

Group Information: Members of the International Skin Imaging Collaboration Melanoma Project Working Groups are listed at the end of this article.

Corresponding Author: Ashfaq A. Marghoob, MD, Dermatology Service, Memorial Sloan Kettering Cancer Center, 800 Veterans Memorial Hwy, Hauppauge, NY 11788 (marghooa@mskcc.org).

Published Online: May 13, 2015.

doi:10.1001/jamadermatol.2015.32.

Conflict of Interest Disclosures: None reported.

The International Skin Imaging Collaboration (ISIC) Melanoma Project Working Groups: The Technique Working Group members were $\mathrm{H}$. Peter Soyer, MD, Dermatology Research Centre, The University of Queensland, School of Medicine, Translational Research Institute, Brisbane, Australia (coleader); Clara Curiel, MD, University of Arizona Cancer Center and Dermatology Section, University of Arizona, Tucson (coleader); Dennis DaSilva, BS Canfield Scientific Inc, Fairfield, New Jersey; Whitney A. High, MD, JD, MEng, University of
Colorado, Aurora; Lynne H. Morrison, MD, Oregon Health and Science University, Portland; and Jeb Zirato, BS, University of Arizona Cancer Center, Tucson.

The Terminology Working Group members were Harald Kittler, MD, Department of Dermatology, Medical University of Vienna, Austria (leader); Giuseppe Argenziano, MD, Arcispedale S. Maria Nuova, Reggio Emilia, Italy; Ralph P. Braun, MD, University Hospital of Zurich, Zurich, Switzerland; Holger Haenssle, MD, University Medical Centre, Goettingen, Germany; Scott W. Menzies, MD, University of Sydney at the Sydney Cancer Centre, Sydney, Australia; Susanna Puig, MD, Hospital Clinic of Barcelona, Barcelona, Spain; Alon Scope, MD Sheba Medical Center and Sackler Faculty of Medicine, Tel Aviv, Israel; Wilhelm Stolz, MD, Munich Municipal Hospital Group, Munich, Germany; Luc Thomas, MD, PhD, University Claude Bernard Lyon and Cancer Research Center of Lyon, Lyon, France; and Iris Zalaudek, MD, Medical University of Graz, Graz, Austria.

The Technology Working Group members were Josep Malvehy, MD, Hospital Clinic of Barcelona, Institut d'Investigacions Biomèdiques August Pi i Sunyer, and Centro de Investigación Biomédica en Red de Enfermedades Raras, Barcelona, Spain (leader); Mani Abedini, PhD, Qiang Chen, PhD, Rahil Garnavi, PhD, and Xingzhi Sun, PhD, IBM Research Australia, Melbourne; Doug Canfield, BS, Canfield Scientific Inc, Fairfield, New Jersey; Noel C. F. Codella, PhD, IBM T.J. Watson Research Center, Yorktown Heights, New York; Rafael Garcia, PhD, and Josep Quintana, PhD, University of Girona, Spain; Constantino Grana, PhD, and Giovanni Pellacani, MD, University of Modena and Reggio Emilia, Modena, Italy; Miron Josipovic, Derma Medical Systems, Vienna, Austria; Peter Klar, Visiomed AG, Bielefeld, Germany; Andreas Mayer, FotoFinder Systems, Inc, Bad Birnbach, Germany; Scott W. Menzies, MD, University of Sydney at the Sydney Cancer Centre, Sydney, Australia; Matthew A. Molenda, MD, ProMedica, Toledo, Ohio; Nizar Mullani, BSc, 3GEN, LLC, and TransLite, LLC, Dana Point, California; Susanna Puig, MD, Hospital Clinic of Barcelona, Barcelona, Spain; Victor Skladnev, BSc, MEng, AIMEDICS Pty Ltd, Eveleigh, New South Wales, Australia; and William V. Stoecker, MD, University of Missouri Health System, Rolla. The Image Archive member was Rainer HoffmanWellenhof, MD, Department of Dermatology, Research Centre for Teledermatology, Prevention and New Innovative Diagnostic Procedures in 
Dermato-Oncology, Medical University of Graz, Graz, Austria (director).

Additional Contributions: Drs Soyer and Curiel contributed to this editorial on behalf of the Technique Working Group; Dr Kittler, the Terminology Working Group; Dr Malvehy, the Technology Working Group; and Dr HoffmanWellenhof, the Image Archive. None were financially compensated.

\section{REFERENCES}

1. Quigley EA, Tokay BA, Jewell ST, Marchetti MA, Halpern AC. Technology and technique standards for camera-acquired digital dermatologic images: a clinical review [published online May 13, 2015]. JAMA Dermatol. doi.10.1001/jamadermatol.2015.33.

2. Krupinski E, Burdick A, Pak H, et al. American Telemedicine Association's practice guidelines for teledermatology. Telemed J E Health. 2008;14(3): 289-302.

3. Madden BC. A proposal for Working Group 19: dermatologic standards: DICOM whitepaper. http://medical.nema.org/dicom/minutes/WG-06 /2009/2009-08-24/DICOM-Dermatology -whitepaper.doc. Published August 14, 2009. Accessed November 24, 2013.

4. International Health Terminology Standards Development Organisation.
SNOWMED CT. http://ihtsdo.org/snomed-ct/. Accessed November 26, 2014.

5. Publications and standards. Institute of Electrical and Electronics Engineers (IEEE) website. https:

//www.ieee.org/publications_standards/index.html. Accessed November 26, 2014.

6. Studies. International Dermoscopy Society website. http://www.dermoscopy-ids.org/index.php /studies. Accessed November 26, 2014

7. ISIC: Melanoma Project. International Society for Digital Imaging of the Skin website. http://www.isdis .net/index.php/isic-project. Accessed May 11, 2015.

\section{Erythematotelangiectatic Rosacea and Telangiectatic Photoaging Same, Separate, and/or Sequential?}

Jonathan K. Wilkin, MD

In 1994, I described rosacea as a cutaneous and ocular vascular disease, ${ }^{1}$ which was based on premises the most compelling of which was that patients with severe flushing due to systemic disease often had $\leftarrow$ Related article page 825 rapidly progressive rosacea, including ocular rosacea, facial telangiectasia, and phymatous changes. The earliest stages of rosacea were proposed to have an inflamed superficial vasculature and lowgrade sterile superficial dermal cellulitis due to recognized provocative factors, such as local irritants, temperature extremes, wind, and flushing reactions. Subsequently, I have sought articles adding molecular details to my mental picture of this vascular pathogenesis of rosacea, and the evidentiary harvest has been abundant.

Steinhoff et $\mathrm{al}^{2,3}$ demonstrated that (1) transient receptor potential vanilloid subfamily (TRPV) receptors are activated by typical rosacea trigger factors, such as heat, capsaicin, and inflammatory mediators, suggesting that flushing from these trigger factors may be via TRPV-positive blood vessels and mast cells; (2) erythematotelangiectatic rosacea (ETR), papulopustular rosacea (PPR) and phymatous rosacea may have different TRPV subtype profiles, with intergrades between ETR and PPR suggesting the possibility of a "march" in some patients with ETR toward PPR; and (3) pituitary adenylate cyclaseactivating polypeptide, a potent vasodilator, is upregulated 20 to 30 times in early ETR. Clearly, inflammatory rosacea cannot stand as a synonym exclusively for PPR, given this evidence for inflammatory events in ETR.

Another factor postulated to possibly contribute to the vascular pathogenesis of rosacea was actinic damage, ${ }^{1}$ especially to the elastin network as a low-resistance pathway through the interstitium along which macromolecules pass to the lymphatic system. ${ }^{4,5}$ Actinic damage here might lead to low-grade superficial dermal lymphatic failure in the rosacea distribution. ${ }^{1}$
Moreover, photodistributed, mostly vasodilator drug-related telangiectasia underscores the potential role for actinic exposure in the pathogenesis of telangiectasia. ${ }^{6}$ Yano et $\mathrm{al}^{7}$ and Kajiya et $\mathrm{al},{ }^{8}$ in exploring the mechanisms of acute UV-B-induced angiogenesis and photodamage in human skin, demonstrated the consequent epidermal hyperplasia, infiltration of elastinproducing neutrophils, and elastin fiber damage, along with a significant increase in both vascular density and vessel size, features consistent with the proposed pathogenesis of ETR ${ }^{1}$ as well as the cutaneous signs of telangiectatic photoaging (TP), as described by Helfrich et al. ${ }^{9}$ There are now plausible molecular links for clinically well-recognized rosacea triggers, including actinic damage, with the proposed inflammatory vascular pathogenesis of rosacea, beginning with ETR. ${ }^{1}$

However, not everyone agrees that rosacea begins with ETR or that ETR is even a subtype of rosacea. Helfrich et $\mathrm{al}^{9}$ remind us that ETR is probably the most disputed subtype of rosacea, with some authors arguing that it is merely photodamage or, at least, difficult to distinguish from photodamage. This is a curious twist given that rosacea was first recognized as a distinct facial dermatosis separate from common acne (acne vulgaris) by virtue of its rosy hue (acne rosacea). ${ }^{10}$ Helfrich et al find that ETR is, in fact, distinguishable from TP.

This study by Helfrich et $\mathrm{al}^{9}$ is pharmacotherapeutically timely, given the current state of drug product development for ETR. Perhaps their distinction between ETR and TP will enable the physician to better pair treatments with specific dermatoses. Even in the era before the approval of drug products for ETR, identification of erythema and telangiectasia had a role. The occasional patient would have dramatic reduction in facial erythema when given systemic antibiotic and antiflushing therapy. Among this minority, fewer still would experience PERT (posterythema-revealed telangiectasia), ${ }^{1}$ the emergence of preexisting telangiectasia from a receding intense erythema. 